1996

\title{
Internal waves in xenon near the critical point
}

Robert F. Berg

M.J. Lyell

G. B. McFadden

Ronald G. Rehm

Follow this and additional works at: https://researchrepository.wvu.edu/faculty_publications

\section{Digital Commons Citation}

Berg, Robert F.; Lyell, M. J.; McFadden, G. B.; and Rehm, Ronald G., "Internal waves in xenon near the critical point" (1996). Faculty Scholarship. 199.

https://researchrepository.wvu.edu/faculty_publications/199

This Article is brought to you for free and open access by The Research Repository @ WVU. It has been accepted for inclusion in Faculty Scholarship by an authorized administrator of The Research Repository @ WVU. For more information, please contact ian.harmon@mail.wvu.edu. 


\section{Internal waves in xenon near the critical point}

Robert F. Berg, M. J. Lyell, G. B. McFadden, and Ronald G. Rehm

Citation: Physics of Fluids 8, 1464 (1996); doi: 10.1063/1.868923

View online: https://doi.org/10.1063/1.868923

View Table of Contents: http://aip.scitation.org/toc/phf/8/6

Published by the American Institute of Physics

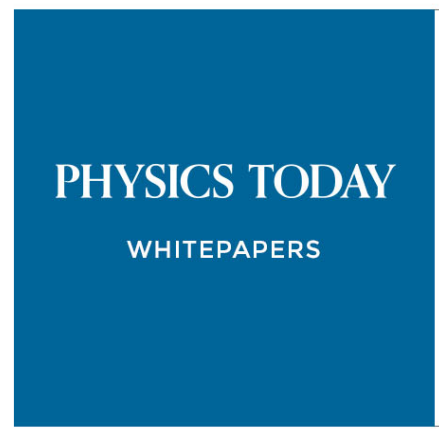




\title{
Internal waves in xenon near the critical point
}

\author{
Robert F. Berg, ${ }^{\text {a) }}$ M. J. Lyell, ${ }^{\text {b) }}$ G. B. McFadden, ${ }^{\text {c) }}$ and Ronald G. Rehm ${ }^{\text {d) }}$ \\ National Institute of Standards and Technology, Gaithersburg, Maryland 20899
}

(Received 11 September 1995; accepted 13 February 1996)

\begin{abstract}
Just above the liquid-vapor critical point, a fluid's large compressibility causes a stable stratification in which the density varies by as much as $10 \%$ in $1 \mathrm{~cm}$. This stratification supports internal gravity waves which we observed with an oscillator immersed in a near-critical xenon sample. We found the number and frequencies of the observable modes depended on the sample cell's orientation, with only two modes seen in the horizontal cell. The frequencies of the two modes had different temperature dependences: with decreasing temperature, the higher frequency increased monotonically from 0.7 to $2.8 \mathrm{~Hz}$, but the lower frequency varied nonmonotonically, with a maximum of $1.0 \mathrm{~Hz}$ at $20 \mathrm{mK}$ above the critical temperature. These temperature dependences continued to $20 \mathrm{mK}$ below the critical temperature, where the xenon was separated into liquid and vapor phases. We calculated these two frequencies by solving the eigenvalue problem of internal waves in a box containing a stratified fluid. The fluid's density profile was obtained from xenon's equation of state. The calculated and measured frequencies agree to within 15\%. Analytical calculations based on simple approximations of the density profile provide insight into the observed temperature dependences. (c) 1996 American Institute of Physics. [S1070-6631(96)00806-5]
\end{abstract}

\section{INTRODUCTION}

Just above the liquid-vapor critical point, a fluid's large compressibility causes a stable vertical stratification in which the density varies by as much as $10 \%$ in $1 \mathrm{~cm}$. In a closed container, this unusual density profile can support internal gravity waves that are sustained by the potential energy generated by vertical displacement of the fluid elements, and whose modes are reminiscent of the "sloshing" modes of a cup of water. Such gravity-induced stratification is a special case of a continuous density profile, and it is interesting for several reasons. First, for all pure fluids at the liquid-vapor critical point, the density profile has a universal sigmoid shape (curved like the letter S). Second, the density profile can be varied from sigmoid to linear simply by raising the sample's temperature. Third, the stratification is a controlled, stable equilibrium state. In contrast, the salt-water layers used in other laboratory studies of internal waves relax via diffusion.

Descriptions of internal-wave motion are frequently included in textbooks on hydrodynamics, ${ }^{1-5}$ and more detailed discussions can be found in specialized books. ${ }^{6-9}$ Internalwave modes are important for the understanding of fluid motions in any stratified fluid, such as the atmosphere, the ocean, lakes, stellar interiors, and even the air in buildings naturally stratified by heat or accidentally stratified by fires. ${ }^{10}$ Experimentalists have previously remarked on the existence of internal-wave modes near the critical point. ${ }^{11}$ Only a few papers have been published on this subject, mostly in Soviet journals. ${ }^{12-14}$ These papers were concerned with exploratory questions such as the existence of gravitational waves or the

\footnotetext{
a) Thermophysics Division, Chemical Science and Technology Laboratory.

b) Permanent address: Mechanical and Aerospace Engineering Department, West Virginia University, Morgantown, West Virginia 26506.

c) Applied and Computational Mathematics Division.

${ }^{d)}$ Computing and Applied Mathematics Laboratory.
}

dispersion relation at the critical temperature $T_{c}$. Thus they could not help us in identifying the observed internal-wave modes or in measuring and calculating their frequencies.

Figure 1 shows the form of the density profile $\rho(z)$ near the critical point of xenon. At temperatures far above $T_{c}$, $\rho(z)$ is approximately linear in the height $z$ because the fluid's compressibility is independent of $z$. As discussed later, this linear profile can be approximated by an exponential profile, thus allowing a closed-form solution for the internalwave modes in a box. However, at temperatures just above $T_{c}$, the profile is strongly nonlinear because there the fluid's compressibility is a strong function of density as well as temperature. At $T_{c}$, the slope $d \rho / d z$ diverges at the height where the density equals the critical density $\rho_{c}$. Below $T_{c}$, a sharp interface, where the density changes discontinuously, divides the liquid and vapor regions. At temperatures far below $T_{c}$, the variations of density within each region are small compared to the difference between the regions. Approximation of the density profile by two discrete densities allows a closed-form solution.

We observed internal waves with an oscillator immersed in a near-critical xenon sample. The number and frequencies of the observable modes depended on the sample cell's orientation, with only two modes seen in the horizontal cell. The frequencies of the two modes had different temperature dependences: with decreasing temperature, the higher frequency increased monotonically from $0.7 \mathrm{~Hz}$ to $2.8 \mathrm{~Hz}$, but the lower frequency varied nonmonotonically, with a maximum of $1.0 \mathrm{~Hz}$ at $20 \mathrm{mK}$ above the critical temperature. (See Figure 2.) We calculated these two frequencies by solving the eigenvalue problem of internal waves in a box containing stratified fluid. The fluid's density profile was obtained from xenon's equation of state. The calculated and measured frequencies agree to within $15 \%$.

When the cell's orientation was changed by $90^{\circ}$, the oscillator coupled to as many as five modes of the xenon. 


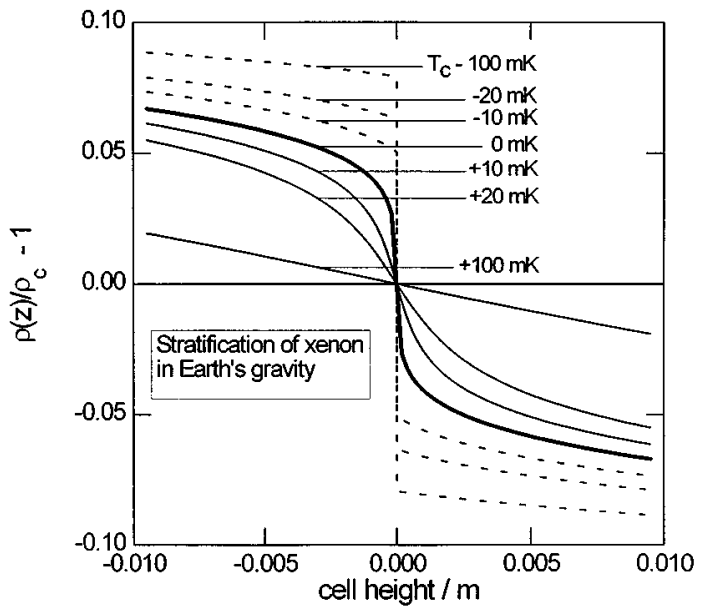

FIG. 1. The density profile of the xenon sample calculated by Eqs. (1) at various reduced temperatures. Far above $T_{c}$, the height dependence is weak and approximately linear. At $T_{c}$, the density gradient is infinite at the middle height, where $\rho=\rho_{c}$. Far below $T_{c}$, the xenon separates into liquid and vapor layers, each of nearly constant density.

Again, our calculated frequencies were consistent with the temperature-dependent range of the observed frequencies.

Our measurements were motivated by the need to characterize accurately a viscometer intended to measure the viscosity of xenon near its liquid-vapor critical point. ${ }^{15,16}$ The viscometer consisted of an overdamped, oscillating screen immersed in the xenon, and changes in viscosity could be inferred from changes of the oscillator's transfer function. The transfer function is the frequency-dependent ratio of the oscillator's displacement to its applied torque. Close to $T_{c}$, the stratification caused by Earth's gravity limited the viscosity measurement in two ways. First, the interesting region having densities near the critical density $\rho_{c}$ narrowed to a thin layer near the sample's midplane. This limited the precision of the measurement of the critical point enhancement of viscosity. Second, the stratification supported internal

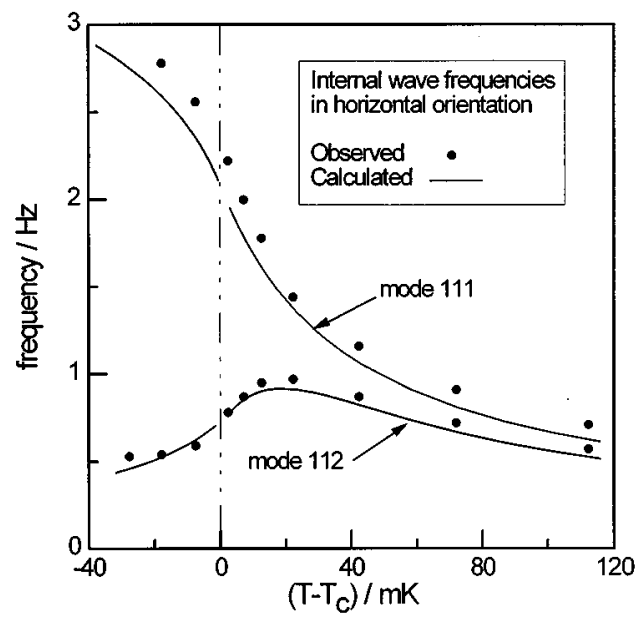

FIG. 2. Internal wave frequencies, measured (circles) and calculated (lines). The cell's axis was horizontal and the screen's torsion axis was vertical, so that the screen's motion was horizontal. The numerical calculations used the actual profiles derived from xenon's equation of state.

\section{CVX Viscometer}

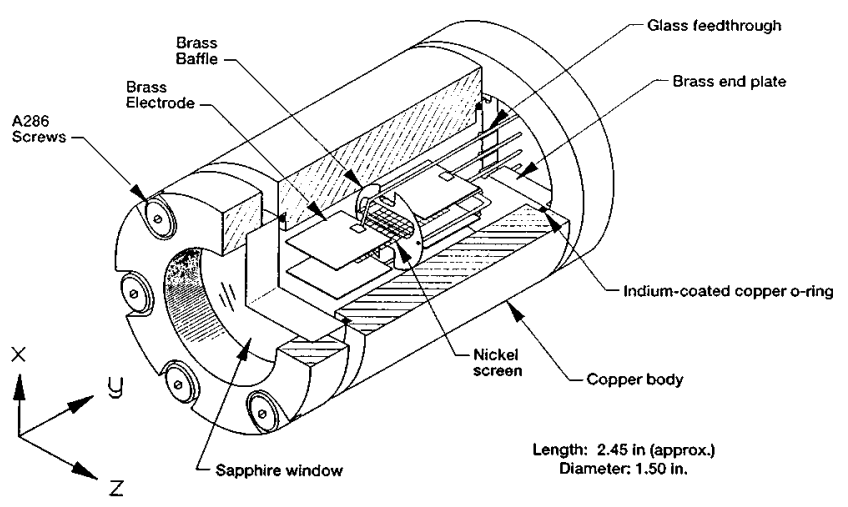

(a)

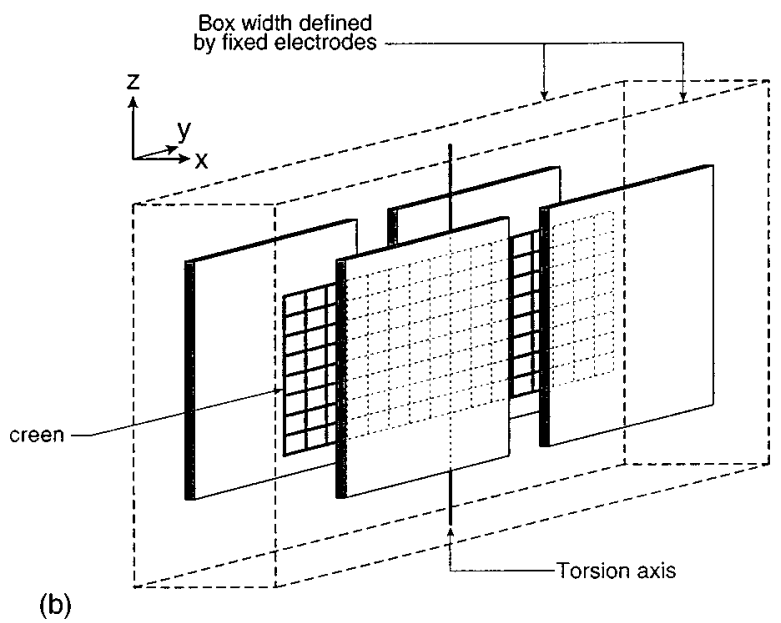

FIG. 3. (a) Schematic diagram of the viscometer to be used in the Critical Viscosity Experiment (CVX). The brass baffle, introduced to reduce the amplitude of the internal waves, was not present during these measurements. During the measurements made with cell's axis horizontal, the cell was rotated so that gravity was along the $z$-axis. (b) The box used to calculate the internal wave frequencies. The box width $a$ was chosen as the distance between the front pair of fixed electrodes. The box length $b$ was defined as the cell's length, and the box height $2 L$ as the cell's height. This approximation ignored the various supporting wire struts, which occupied a small fraction of the cell's cross-section, and the oscillating screen itself, because of its small mass and weak mechanical compliance. The approximation also assumed the opposing pairs of electrodes acted as rigid, vertical walls spanning the cell's height and length. In reality, each pair occupied only $1 / 3$ of the area $b c$. However, the pairs overlapped all of the screen except for a small portion near the torsion axis, where the screen's motion was small. Gravity is along the (vertical) $z$-axis.

wave modes within the viscometer's bandwidth of operation. Because these internal-wave modes were not included in the model of the viscometer, they limited the measurement's accuracy.

The viscometer was designed for ultimate use in the Space Shuttle's microgravity, where the effect of internal waves will be negligible. The xenon was contained within a simple cylinder; however, the internal geometry was complicated by electrodes and the oscillator itself. (See Figure 3.) The calculation of the frequencies of the internal-wave 
modes relied on a simple approximation of the container's internal geometry.

Fortuitously, the viscometer had several advantages for studying internal-wave modes. First, the viscometer was sensitive to three-dimensional internal-wave modes because the oscillator had both a sufficiently low mass and a low resonance frequency so that it was sensitive to weak hydrodynamic forces, and because the oscillator spanned at least half the length of the sample cell. In contrast, conventional techniques for flow visualization, such as schlieren imaging or the use of tracer particles, are best suited for twodimensional flows only. The second advantage was the viscometer's internal symmetry, which restricted the oscillator from coupling to most of the internal-wave modes. This restriction was useful due to the small spacing between modes. For example, even with the three modal indices restricted to only 0,1 , or 2 , the frequencies calculated for 9 of the 16 possible modes can fall within a range of only $6 \%$. Without the coupling restriction, the overlap of many modes would have complicated the interpretation of the measurements.

In the following, after briefly explaining the nature of the density profile, we will describe our measurements of the internal wave frequencies. Then we will describe the computational techniques that we used to obtain these frequencies from the equations of fluid mechanics, the sample cell's geometry, and xenon's equation of state. The measured and calculated frequencies will then be compared.

\section{THE DENSITY PROFILE NEAR THE CRITICAL POINT}

We calculated the sample's density profile $\rho(z)$ from xenon's equation of state. Near the liquid-vapor critical point, the divergences of thermodynamic derivatives such as compressibility and heat capacity cause conventional equations of state to fail. Thus, we used Ho and Lister's 17 "restricted cubic model" which accounts for such divergences in a natural manner. This scaled equation of state, summarized by Moldover et al. ${ }^{18}$ uses the parametric variables $r$ and $\theta$, which are defined in terms of the temperature $T$ and density $\rho$ (or the reduced variables $\Delta T^{*}$ and $\Delta \rho^{*}$ ) by

$$
\begin{aligned}
& \Delta T^{*} \equiv \frac{\left(T-T_{c}\right)}{T_{c}}=r\left(1-b^{2} \theta^{2}\right), \\
& \Delta \rho^{*} \equiv \frac{\left(\rho-\rho_{c}\right)}{\rho_{c}}=k r^{\beta} \theta\left(1+c \theta^{2}\right), \\
& \Delta \mu^{*} \equiv\left(\frac{\rho_{c}}{P_{c}}\right)\left[\mu(\rho, T)-\mu\left(\rho_{c}, T\right)\right]=a r^{\beta \delta}\left(1-\theta^{2}\right) .
\end{aligned}
$$

The variable $\theta$ can range from -1 to +1 , with $\theta=0$ corresponding to $\rho_{c}$. The last equation defines the reduced chemical potential $\Delta \mu^{*}$. Here $P_{c}$ is the critical pressure. The isothermal density profile $\rho(z)$ can then be determined from the variation of the reduced chemical potential with height, ${ }^{18}$ $\Delta \mu^{*}=-\left(g \rho_{c} / P_{c}\right) z$, where $g$ is the gravitational acceleration and $z \equiv 0$ at $\rho=\rho_{c}$. In the above expressions, the constants $b$ and $c$ and the exponents $\beta$ and $\delta$ are universal for all pure fluids, while the constants $a$ and $k$ are peculiar to xenon. (See Table I.) Compressibility has such a strong diver-
TABLE I. Parameters for xenon's equation of state.

\begin{tabular}{lcc}
\hline \hline$T_{c}$ & 289.7 & $\mathrm{~K}$ \\
$P_{c}$ & 5.840 & $\mathrm{MPa}$ \\
$\rho_{c}$ & 1110 & $\mathrm{~kg} \cdot \mathrm{m}^{3}$ \\
$a$ & 15.4 & \\
$k$ & 0.89 & \\
$B$ & 1.43 & \\
$\beta$ & 0.325 & \\
$\delta$ & 4.815 & \\
$b^{2}$ & 1.277 & \\
$c$ & 0.055 & \\
\hline \hline
\end{tabular}

gence that the critical region parameters can be determined from optical measurements of $\rho(z)$. We used the values $a$ and $k$ determined by Hocken and Moldover. ${ }^{18,19}$ Solution of Eqs. (1) yields the density profile, and profiles calculated for several temperatures near $T_{c}$ are shown in Figure 1. Profiles such as these were used in our numerical calculations of the internal-wave frequencies.

At temperatures below $T_{c}$, the fluid separates into liquid and vapor phases separated by a discontinuous change of density. As shown in Figure 1, the density varies continuously with height within each phase. However, below $T_{c}-20 \mathrm{mK}$, this variation is small compared to the difference between phases, and the densities of the two phases are then given by the restriction $\theta= \pm 1$, so that

$$
\rho=\rho_{c}\left\{1 \pm B\left[\left(\frac{T_{c}-T}{T_{c}}\right)^{\beta}\right]\right\} .
$$

Here, the " + " is used for the denser, liquid phase, and $B \simeq 1.602 k$.

\section{APPARATUS}

The viscometer consisted of an oscillator and its associated electrodes contained within a copper cell whose inner, cylindrical space was $38 \mathrm{~mm}$ long and $19 \mathrm{~mm}$ in diameter. One end of the cylinder was sealed by a sapphire window, and the other end by a brass plate containing five electrical feedthroughs connected to the oscillator and its electrodes.

Figure $3 \mathrm{a}$ is a sketch of the viscometer. The oscillator was constructed by cutting an $8 \times 19 \mathrm{~mm}$ rectangle out of a larger piece of nickel screen while leaving attached two wire extensions that formed the torsion fiber. The screen's crosssection area in the flow direction was small: it consisted of $0.03-\mathrm{mm}$ wide wires spaced $0.85 \mathrm{~mm}$ apart.

The torsion fibers were attached to a stiff yoke with $\mathrm{Pb}-\mathrm{Sn}$ solder. The yoke was centered between four electrodes parallel to the screen, and the complete assembly was sealed into the cell. The fixed electrodes, separated by a 7.6 $\mathrm{mm}$ gap, were connected electrically into diagonally opposite pairs. In vacuum, the lowest resonance frequency of the oscillator was the torsion mode at $11 \mathrm{~Hz}$, which was antisymmetric about the torsion axis. The resonance frequency of the next lowest mode was much higher, above $50 \mathrm{~Hz}$, and this mode was symmetric about the torsion axis. Due to both the large difference in these two frequencies and the symmetry of the driving electrodes, only the torsion mode was excited in the present measurements. 
After characterization of the oscillator in vacuum, xenon was loaded into the viscometer cell. The loading was adjusted at a temperature just below $T_{c}$ until the liquid-vapor meniscus was at the cell's middle height, so that the average density in the cell was within $0.2 \%$ of the critical density $\rho_{c}$. Observation of the appearance and disappearance of the meniscus determined $T_{c}$ to within $1 \mathrm{mK}$.

After loading, the cell was placed into a thermostat consisting of three independently-controlled, concentric aluminum shells. In this environment, the cell's temperature was measured to be stable to within $0.03 \mathrm{mK}$ over several hours, and the maximum temperature difference across the cell was calculated to be less than $0.2 \mu \mathrm{K}$.

A commercial spectrum analyzer generated oscillating source voltages which, after modification, were used to apply torques to the oscillator. The modification compensated for the quadratic dependence of torque on the voltage between the electrode pairs and the screen. The source voltage was first added to two dc bias voltages of opposite sign. The biased sums were then amplified by square root amplifiers and the resulting voltages were applied to the diagonally opposed electrode pairs.

The oscillating screen and the fixed electrodes also formed a capacitance bridge that was operated at $10 \mathrm{kHz}$ to detect the screen's displacement. The bridge's signal was detected by a lock-in amplifier whose time constant was set sufficiently low, from 0.04 to $0.4 \mathrm{~s}$, to avoid attenuation of the detected motion. The amplifier's output was proportional to the difference between the capacitance of each pair of electrodes and the screen; thus it was a nearly linear function of the screen's displacement. The amplifier's output was continuously measured by the spectrum analyzer.

Further details may be found in References 15 and 16.

\section{MEASUREMENT OF THE INTERNAL WAVE FREQUENCIES}

Our measurement technique assumed the internal-wave mode was a massive, high- $Q$ oscillator coupled weakly to the low-mass oscillating screen. The expected $Q$ of an internal wave mode was estimated to be $R / \delta$, where $R>3 \mathrm{~mm}$ is a length typical of the sample cell's interior, and $\delta$ is the viscous penetration length. At the typical internal-wave frequency of $\omega / 2 \pi=1 \mathrm{~Hz}$,

$$
\begin{aligned}
\delta & =\sqrt{\frac{2 \eta}{\rho_{c} \omega}} \\
& =\sqrt{\frac{(2)\left(5 \times 10^{-5} \mathrm{~Pa} \cdot \mathrm{s}\right)}{\left(1100 \mathrm{~kg} \cdot \mathrm{m}^{-3}\right)\left(2 \pi \cdot 1 \mathrm{~s}^{-1}\right)}}=0.12 \mathrm{~mm},
\end{aligned}
$$

where $\eta$ is xenon's viscosity. Thus $Q \approx R / \delta>25$ was expected. Although the observed $Q$ 's were less than 25, they were still sufficiently large for the technique to succeed.

To bring the fluid into steady oscillations, the screen was driven at a frequency $f_{\text {drive }}$ for at least 10 cycles. Then the drive was turned off, and the screen's residual motion was recorded. If $f_{\text {drive }}$ was near an internal-wave frequency, subsequent transient oscillations were visible, and the frequency

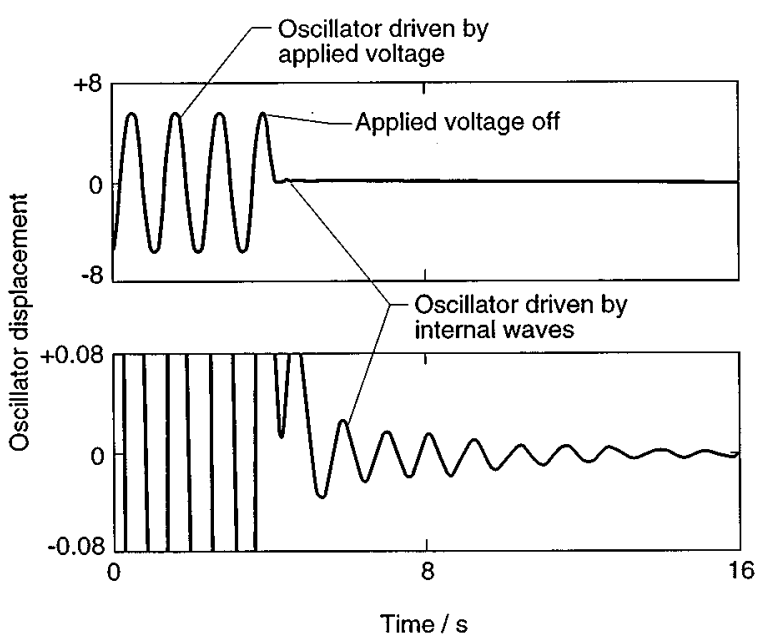

FIG. 4. Example of an internal wave frequency measurement. (a) The oscillator's displacement shown during and after application of an oscillating torque at $0.875 \mathrm{~Hz}$. (b) The oscillator's displacement during the same interval, magnified by 100 .

of the internal wave was assigned after examination of the transient waveform's spectrum. Figure 4 shows an example of a particularly strong internal-wave mode.

To ensure equilibrium of the density profile, we waited at least 4 hours after changing the temperature. Due to the fluid's slow thermal diffusion, such a wait is typical for centimeter-sized critical point samples. Then a series of drive frequencies was used to search for internal-wave modes. Typically, this search covered the range from 0.5 to $2.0 \mathrm{~Hz}$ in $0.1 \mathrm{~Hz}$ steps. The oscillator's small amplitude did not disrupt the stratification, as evidenced by the reproducibility of successive searches made at the same temperature. Thus 15 drive frequencies could be tested in as many minutes. Searches to as low as $0.3 \mathrm{~Hz}$ or as high as $20 \mathrm{~Hz}$ found no modes other than those reported here.

Two sets of measurements were made. The first set, listed in Table II, was made with the cell's axis horizontal and the oscillator's torsion axis vertical. The second set was made with the cell's axis vertical, so that the oscillator's torsion axis was horizontal.

TABLE II. Experimental and calculated frequencies in $\mathrm{Hz}$ for the horizontal cell.

\begin{tabular}{rcccc}
\hline \hline$\left(T-T_{c}\right) / \mathrm{mK}$ & exp. (111) & exp. (112) & calc. (111) & calc. (112) \\
\hline-27.6 & - & 0.53 & 2.753 & 0.460 \\
-17.8 & 2.78 & 0.54 & 2.593 & 0.526 \\
-7.5 & 2.56 & 0.59 & 2.358 & 0.623 \\
2.4 & 2.22 & 0.78 & 1.979 & 0.772 \\
7.2 & 2.00 & 0.87 & 1.786 & 0.853 \\
12.6 & 1.78 & 0.95 & 1.618 & 0.901 \\
22.3 & 1.44 & 0.97 & 1.377 & 0.913 \\
42.4 & 1.16 & 0.87 & 1.066 & 0.823 \\
72.0 & 0.91 & 0.72 & 0.810 & 0.667 \\
112.4 & 0.71 & 0.57 & 0.624 & 0.523 \\
\hline \hline
\end{tabular}




\section{THEORETICAL FORMULATION}

Both buoyancy and acoustic forces contribute to the oscillations of a fluid element. The associated Brunt-Väisälä frequency $N(z)$ is, at each height $z$, the local frequency with which an adiabatic, inviscid fluid element oscillates. ${ }^{9}$ It can be expressed as the sum of two terms,

$$
N^{2}(z)=-\left(\frac{g}{\rho} \frac{d \rho}{d z}+\frac{g^{2}}{c_{0}^{2}}\right),
$$

where $g$ is the gravitational acceleration, $\rho(z)$ is the local fluid density, and $c_{0}(z)$ is the local speed of sound. If the $g^{2} / c_{0}^{2}$ term is small relative to the first term, then, according to Ref. 9, the coupling between internal waves and acoustic waves is negligible.

We verified $(g / \rho) d \rho / d z \gg g^{2} / c_{0}^{2}$ by numerically calculating both terms in the stratified xenon. The speed of sound was calculated from thermodynamic principles and parameter values specific to xenon. ${ }^{20,21}$ The density derivative profile was calculated from Eqs. (1). At all heights in the sample, $(g / \rho) d \rho / d z$ was at least two orders of magnitude greater than $g^{2} / c_{0}^{2}$, and thus compressibility effects on the wave motion could be neglected.

The development of this section proceeds as follows: the set of partial differential equations governing the fluid dynamic behavior is introduced and linearized for small velocity. The boundary conditions, together with various approximations, are used to generate an ordinary differential equation in the amplitude of the vertical velocity which contains the frequency, an unknown parameter. This differential equation and its boundary conditions form an eigenvalue problem. This problem is solved for four classes of density profiles. The special case of the exponential density profile is solved first. Next, the general problem for $T>T_{c}$, where xenon is stratified in a single phase, is solved using two independent numerical methods. Then, the general problem for $T<T_{c}$, where an interface separates the xenon into stratified liquid and vapor phases, is addressed numerically. Finally, the special case where an interface separates two fluids, each of constant density, is solved.

\section{A. Eigenvalue problem development}

The derivation of the equation for the vertical component of the perturbation velocity follows, for example, Ref. 8. The analysis is for an adiabatic, incompressible, and inviscid fluid. The governing equations are given by

$$
\begin{aligned}
& \boldsymbol{\nabla} \cdot \mathbf{u}=0, \\
& \rho\left(\frac{\partial \mathbf{u}}{\partial t}+\mathbf{u} \cdot \boldsymbol{\nabla u}\right)=-\boldsymbol{\nabla} p-\rho g \hat{\mathbf{e}}_{z}, \\
& \frac{\partial \rho}{\partial t}+\mathbf{u} \cdot \boldsymbol{\nabla} \rho=0,
\end{aligned}
$$

where $\mathbf{u}=(u, v, w)$ and $p$ denote the velocity and pressure fields, respectively. Here $\hat{\mathbf{e}}_{z}$ is a unit vector in the $z$ direction (anti-parallel to gravity). Perturbation about the state of zero velocity and hydrostatic equilibrium, followed by linearization, yields the perturbation partial differential equations

$$
\begin{aligned}
& \boldsymbol{\nabla} \cdot \mathbf{u}^{(1)}=0, \\
& \rho^{(0)} \frac{\partial \mathbf{u}^{(1)}}{\partial t}=-\boldsymbol{\nabla} p^{(1)}-\boldsymbol{\rho}^{(1)} g \hat{\mathbf{e}}_{z}, \\
& \frac{\partial \boldsymbol{\rho}^{(1)}}{\partial t}+w^{(1)} \frac{d \boldsymbol{\rho}^{(0)}}{d z}=0,
\end{aligned}
$$

where $\rho^{(0)}$ is the density of the unperturbed base state, and the superscript "(1)" denotes the deviation of quantities from equilibrium.

The xenon-filled viscometer is modeled as a rectangular box defined by $0 \leqslant x \leqslant a, 0 \leqslant y \leqslant b$, and $-L \leqslant z \leqslant L$. (The choices of the box's dimensions will be discussed in the Results section.) The normal component of velocity at the walls must be zero. This implies that at $x=0$ and at $x=a$, $u^{(1)}=0$, while at $y=0$ and at $y=b, v^{(1)}=0$. This suggests that the form of the perturbation field is

$$
\begin{aligned}
& u^{(1)}(x, y, z, t)=\hat{u}(z) \sin \left(q_{x} x\right) \cos \left(q_{y} y\right) e^{i \omega t}, \\
& v^{(1)}(x, y, z, t)=\hat{v}(z) \cos \left(q_{x} x\right) \sin \left(q_{y} y\right) e^{i \omega t}, \\
& w^{(1)}(x, y, z, t)=\hat{w}(z) \cos \left(q_{x} x\right) \cos \left(q_{y} y\right) e^{i \omega t}, \\
& p^{(1)}(x, y, z, t)=\hat{p}(z) \cos \left(q_{x} x\right) \cos \left(q_{y} y\right) e^{i \omega t}, \\
& \rho^{(1)}(x, y, z, t)=\hat{\rho}(z) \cos \left(q_{x} x\right) \cos \left(q_{y} y\right) e^{i \omega t},
\end{aligned}
$$

where $q_{x} \equiv \pi j / a$ and $q_{y} \equiv \pi k / b$ are the wave numbers in the $x$ and $y$ directions and the integers $j$ and $k$ are the corresponding mode indices. Substitution of Eqs. (7) into Eqs. (6) yields a set of ordinary differential equations for the perturbation eigenfunctions, which are the $z$-dependent quantities indicated by the caret. Upon manipulation, these equations can be combined to yield a single equation for $\hat{w}$, which is

$$
\frac{d^{2} \hat{w}}{d z^{2}}-\frac{N^{2}}{g} \frac{d \hat{w}}{d z}-q^{2}\left(1-\frac{N^{2}}{\omega^{2}}\right) \hat{w}=0 .
$$

Here, $q^{2} \equiv q_{x}^{2}+q_{y}^{2}$, and $N^{2}(z) \equiv-\left(g / \rho^{(0)}\right)\left(d \rho^{(0)} / d z\right)$. Equation (8), together with the boundary conditions $\hat{w}(+L)=\hat{w}(-L)=0$, form the eigenvalue problem for the frequency $\omega$.

\section{B. Special case: Exponential density profile, $\boldsymbol{T}_{>} \boldsymbol{T}_{c}$}

Above $T_{C}+80 \mathrm{mK}$, our xenon sample's density profile was nearly linear, which we approximated by the special case of an exponential density profile. An exponential profile has a Brunt-Väisälä frequency $N$ which is independent of height, allowing solution of the flow field equations in closed form. These solutions provided for rapid visualization of the flow field, and they gave an indication of which modes would be likely to couple to the oscillating screen's motion.

The closed-form eigenfunction solution is given by

$$
\hat{w}(z)=\frac{g}{N} \exp \left(\frac{N^{2} z}{2 g}\right) \sin \left[\left(\frac{\pi l}{2}\right)\left(\frac{z}{L}-1\right)\right] .
$$

The dispersion relation is

$$
\omega^{2}=\frac{q^{2} N^{2}}{q^{2}+\left(N^{2} / 2 g\right)^{2}+(\pi l / 2 L)^{2}} .
$$




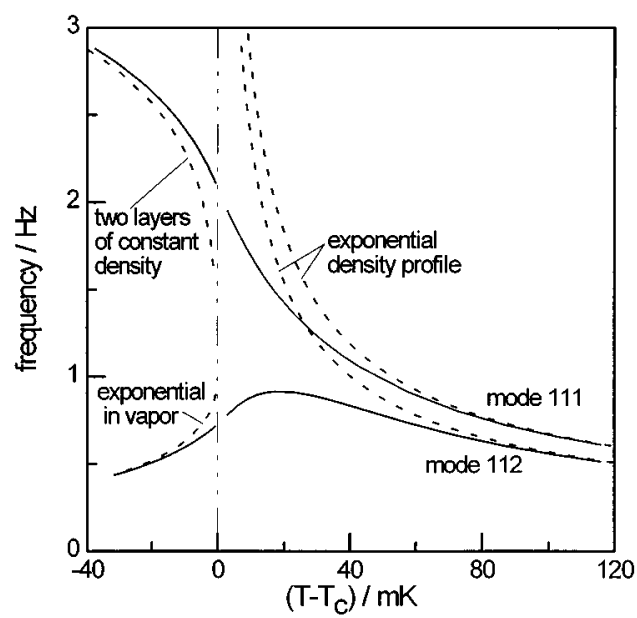

FIG. 5. Comparison between numerically (solid lines) and analytically (dashed lines) computed internal wave frequencies for the horizontal orientation. Above $T_{c}+60 \mathrm{mK}$, the linear density profile can be approximated by an exponential, allowing use of Eq. (10), which matches the numerical results. Below $T_{c}$, the fluid is separated by an interface into liquid and vapor layers. Neglect of the stratification within each layer allows use of Eq. (20), which describes mode (111)'s temperature dependence at temperatures below $T_{c}-5 \mathrm{mK}$. Neglect of the liquid layer entirely and assumption of an exponential density profile within the vapor phase allows the use of Eq. (10) to approximate the temperature dependence of mode (112) below $T_{c}-5$ $\mathrm{mK}$.

Note that $\omega^{2}<N^{2}$, and that $\omega^{2}$ decreases with increasing mode index $l$.

The frequencies calculated for modes $(j k l)=(111)$ and (112) are plotted in Figure 5. The value of $N^{2} / g$ used in Eq. (10) was based on the density gradient at the cell's midplane, in turn calculated from Eqs. (1).

The remaining perturbation velocity components are given by

$$
\hat{u}(z)=\frac{q_{x}}{q^{2}} \frac{d \hat{w}}{d z}, \quad \hat{v}(z)=\frac{q_{y}}{q^{2}} \frac{d \hat{w}}{d z} .
$$

The flow field for modes (111) and (112) are shown in Figures 6 and 7. Although calculated for the case of an exponential density profile, these figures are qualitatively similar to the flow fields of the actual density profiles, even at temperatures close to $T_{c}$. Thus the exponential approximation was useful for deciding which modes coupled to the oscillating screen.

\section{Computational approach: Actual density profile, $T>T_{c}$}

For $T>T_{c}$, the xenon was a single phase fluid whose density profile was not exponential. Therefore, we obtained the eigenfrequencies by numerically solving Eq. (8). It proved convenient to work with a nondimensional equation. Let

$$
z \equiv L Z, \quad \hat{w} \equiv w_{0} W,
$$

where $L$ is the half-height of the box and $w_{0} \equiv(g L)^{1 / 2}$ is a velocity scale. These nondimensionalizations were used in Eq. (8). A pseudospectral collocation method was applied in the standard manner ${ }^{22}$ to the resulting nondimensional equa-
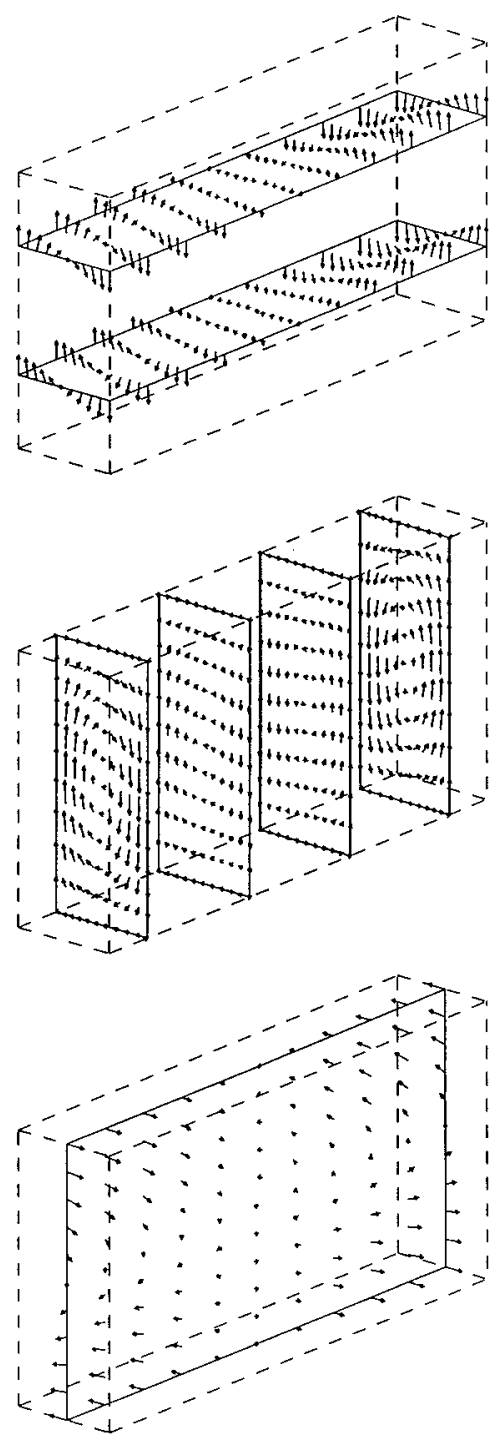

FIG. 6. The flow field of mode (111) calculated by Eqs. (9) and (11) for the special case of an exponential profile with $N^{2}=25 \mathrm{~s}^{-2}$. The coordinates correspond to those of Figure $3 \mathrm{~b}$. In the lowest view, the arrows were lengthened by a factor of 2 relative to those in the upper two views.

tion and its associated boundary conditions. Chebyshev polynomials were used as the expansion functions. It was first necessary to expand the density and density derivative profiles in a Chebyshev representation. We calculated the eigenfrequencies for cases having different density profiles, each associated with a different temperature above $T_{c}$. Closer to $T_{c}$, the density profile's derivative contains a sharp peak. Thus, in order to capture accurately the density and density derivative profiles, it was necessary to employ 900 terms in the Chebyshev expansion for the calculation corresponding to $T=T_{c}+1 \mathrm{mK}$. For $T \geqslant T_{c}+2 \mathrm{mK}, 512$ terms in the Chebyshev expansion were found to be adequate. For $T \geqslant T_{C}+20 \mathrm{mK}, 256$ terms were adequate.

As a check on the frequencies obtained via the collocation approach, an alternate method was used to solve the problem at selected temperatures. This second method, given by Keller, ${ }^{23}$ converted the eigenvalue problem with its ho- 

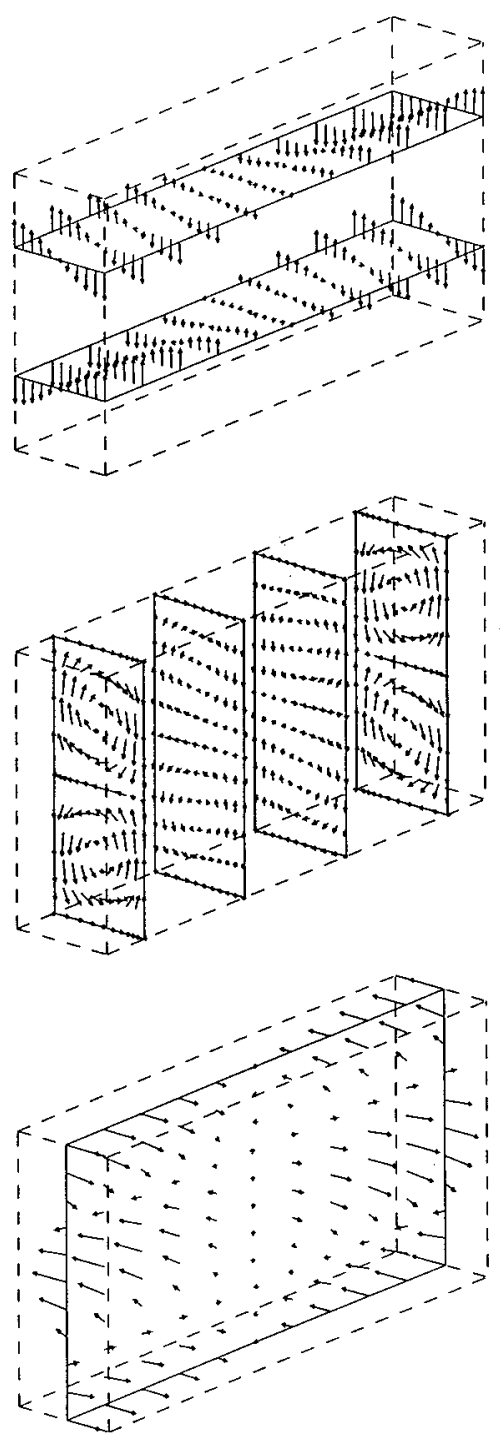

FIG. 7. The flow field of mode (112) calculated by Eqs. (9) and (11) for the special case of an exponential profile with $N^{2}=25 \mathrm{~s}^{-2}$. The coordinates correspond to those of Figure $3 \mathrm{~b}$. In the lowest view, the arrows were lengthened by a factor of 2 relative to those in the upper two views.

mogeneous boundary conditions into an inhomogeneous, two-point boundary value problem with non-constant coefficients. This linear problem was then solved by the SUPORT code. $^{24}$ This numerical code uses superposition of the integrated solutions coupled with a Gram-Schmidt orthonormalization procedure to maintain linear independence of the solutions. Numerical integration of the ordinary differential equation in the SUPORT code is done with a higher-order Adams-type method. In all cases, Keller's method and the pseudospectral collocation method agreed to at least six digits in the eigenvalue.

The frequencies computed for the modes with $(j k l)$ equal to (111) and (112) are plotted in Figures 1 and 5 (for $T>T_{c}$ ). In these computations, the density profiles, calculated from Eqs. (1) using the parameters given in Ref. 18, were the actual profiles expected from xenon's equation of state. Table II contains values calculated at the experimental temperatures.

\section{Computational approach: Actual density profile, $T<T_{c}$}

For $T<T_{c}$, the xenon sample consisted of both vapor and liquid phases separated by an interface. Each of the phases was density stratified. For this case, only a single numerical treatment was employed, again based on the SUPORT code.

It is convenient to work in terms of a perturbed vertical flux $h^{(1)}$, defined as

$$
h_{v}^{(1)} \equiv \rho_{v}^{(0)} w_{v}^{(1)}, \quad h_{l}^{(1)} \equiv \rho_{l}^{(0)} w_{l}^{(1)},
$$

where the vapor and liquid phases are denoted by the subscripts $v$ and $l$, respectively.

Proceeding from the perturbation system of Eqs. (6), a system in $\left(p^{(1)}, h^{(1)}\right)$ can be obtained in each phase. It is given by

$$
\begin{aligned}
& \frac{\partial^{2} h^{(1)}}{\partial t^{2}}+N^{2}(z) h^{(1)}=-\frac{\partial^{2} p^{(1)}}{\partial t \partial z}, \\
& \left(\frac{\partial^{2}}{\partial x^{2}}+\frac{\partial^{2}}{\partial y^{2}}\right) p^{(1)}=\frac{\partial^{2} h^{(1)}}{\partial t \partial z}+\frac{N^{2}(z)}{g} \frac{\partial h^{(1)}}{\partial t} .
\end{aligned}
$$

The boundary conditions on the perturbed vertical flux are $h_{l}^{(1)}(-L)=h_{v}^{(1)}(+L)=0$. The forms of $h^{(1)}$ and $p^{(1)}$ are consistent with the forms of the perturbations given in Eqs. (7).

The interface which separates the vapor and liquid regions is given by

$$
F(x, y, z, t) \equiv z-f(x, y, t)=0 .
$$

In the base state the interface is given by $z=0$, and the form of the interface deflection $f$ is consistent with the forms of the perturbations in Eqs. (7). The pressure and the normal component of the velocity are continuous across the interface. Finally, the kinematic condition $D F / D t=0$ is imposed. After linearization, these boundary conditions at $z=0$ become

$$
\begin{aligned}
& \frac{\hat{h}_{v}}{\rho_{v}^{(0)}}=\frac{\hat{h}_{l}}{\rho_{l}^{(0)}}=i \omega \hat{f}, \\
& \left(\hat{p}_{v}-\hat{p}_{l}\right)=g\left(\rho_{v}^{(0)}-\rho_{l}^{(0)}\right) \hat{f} .
\end{aligned}
$$

Upon approaching $T_{c}$, the effect of surface tension becomes negligible compared to the liquid-vapor density difference. Thus, surface tension is neglected in Eq. (17).

After substitution of the perturbation forms for $h^{(1)}$ and $p^{(1)}$ into Eqs. (14), a linear, coupled system of ordinary differential equations in the perturbation amplitudes $\hat{h}$ and $\hat{p}$ is obtained in each phase. This system is nondimensionalized using the scales given in the previous section, together with the scale $\rho_{c} L g$ for the pressure.

An iterative numerical method is used to determine the frequency in the following manner. The interface deflection $\hat{f}$ is set to a fixed nonzero value. For a given value of the frequency, the system is integrated from $z=-L$ to $z=0$ in 
the liquid region, and from $z=+L$ to $z=0$ in the vapor region, using Eqs. (16) as boundary conditions for $\hat{h}$ at $z=0$. The computed interface pressures from the vapor and liquid regions are then compared, and a root finder is used to vary the frequency iteratively until the force balance (17) is satisfied to a given tolerance.

The eigenfrequencies for modes (111) and (112) obtained for $T<T_{c}$ via this computational scheme are also shown in Figure 2. These calculations used the actual profiles determined from xenon's equation of state. Table II contains values calculated at the experimental temperatures.

\section{E. Special case: Two-layers of constant density, $T<T_{c}$}

The special case in which the density profile consists of a liquid layer and a vapor layer, each of constant density, holds at temperatures far below $T_{c}$. This case is useful for understanding the nature of mode (111) in the horizontal cell. The fluid is assumed to be inviscid and incompressible. The flow in each layer can be taken as irrotational.

The two-layer system is perturbed about the state of zero velocity in the standard manner, ${ }^{25}$ and then linearized. Let the perturbation velocity fields $\mathbf{u}_{v}^{(1)}$ and $\mathbf{u}_{l}^{(1)}$ in each phase be expressed as the gradient of the velocity potentials $\phi_{v}^{(1)}$ and $\phi_{l}^{(1)}$, respectively. Substitution of this form into the conservation of mass condition yields Laplace's equation. At each surface of the container, the normal velocity must be zero. The appropriate form of the perturbation potential in each phase has the form

$$
\phi^{(1)}=\hat{\phi}(z) \cos \left(q_{x} x\right) \cos \left(q_{y} y\right) e^{i \omega t},
$$

where $q_{x} \equiv \pi j / a$ and $q_{y} \equiv \pi k / b$. Substitution of Eq. (18) into Laplace's equation yields an ordinary differential equation in $z$ which has the solutions

$$
\begin{aligned}
& \hat{\phi}_{l}(z)=A_{l} \cosh (q z)+B_{l} \sinh (q z), \\
& \hat{\phi}_{v}(z)=A_{v} \cosh (q z)+B_{v} \sinh (q z),
\end{aligned}
$$

in each phase. As before, $q^{2} \equiv q_{x}^{2}+q_{y}^{2}$. The coefficients $A_{l}$, $B_{l}, A_{v}$, and $B_{v}$ are unknown constants. From the requirement that the vertical velocity be zero at both the top and bottom surfaces of the box, $z= \pm L$, it follows that $B_{l}=A_{l} \tanh (q L)$ and $B_{v}=-A_{v} \tanh (q L)$.

A closed-form dispersion relation for the eigenfrequencies can then be obtained by imposing the interfacial boundary conditions. The dispersion relation takes the form

$$
\omega^{2}=\left[\frac{\rho_{l}^{(0)}-\rho_{v}^{(0)}}{\rho_{l}^{(0)}+\rho_{v}^{(0)}}\right] q g \tanh (q L) .
$$

The horizontal modal dependence enters through the quantity $q$, which depends on the horizontal mode indices $j$ and $k$. Note that because the liquid and vapor phases each have constant density there is no analog of the mode number $l$ in this case; there is a single mode for each value of $q$.

The frequencies resulting from Eq. (20) are plotted in Figure 5. These calculations used Eq. (2) and the parameters from Ref. 18 given in Table I to calculate the densities of the liquid and vapor phases.

\section{RESULTS AND DISCUSSION}

\section{A. Horizontal cell}

Figure 2 shows the results of measurements taken with the cell's axis horizontal and the screen's torsion axis vertical, so that the screen's motion was horizontal. In this orientation, only two modes were observed. Note that the temperature dependence of both modes was continuous through $T_{c}$. The upper mode's temperature dependence was remarkably different from that of the lower mode: the ratio of the two frequencies increased from approximately 1.2 far above $T_{c}$, to 3.2 at $T_{c}$, to 5.2 at $20 \mathrm{mK}$ below $T_{c}$. Also, with decreasing temperature, while the mode at higher frequency increased monotonically from 0.7 to $2.8 \mathrm{~Hz}$, the mode at lower frequency had a maximum of $1.0 \mathrm{~Hz}$ near $T_{c}+20 \mathrm{mK}$. (The experimental values for the horizontal cell are tabulated in Table II.)

Before we could calculate the internal-wave frequencies and compare them with the observed frequencies, we had to approximate the cell's internal volume by a simple shape and identify the observed modes.

As mentioned earlier, the frequency calculations assumed the xenon was contained in a rectangular box. (See Figure 3b.) As explained below, this is a reasonable approximation to the cell's geometry. The box was assigned the dimensions

$$
\begin{gathered}
\text { x-width }=a=7.6 \mathrm{~mm}, \\
\text { y-length }=b=38 \mathrm{~mm}, \\
\text { z-height }=2 L=19 \mathrm{~mm} .
\end{gathered}
$$

The width $a$ was chosen as the distance between the front pair of fixed electrodes. The length $b$ was defined as the cell's length, and the height $2 L$ as the cell's height. This approximation ignored the various supporting 1-mm diameter wires, whose hydrodynamic cross-section was only a small fraction of the cell's cross-section, and the screen itself, because of its small mass and weak mechanical stiffness. The approximation also assumed the opposing pairs of electrodes acted as rigid, vertical walls spanning the cell's height and length. In reality, each pair occupied only $1 / 3$ of the area $2 L b$. However, the pairs overlapped all of the screen except for a small portion near the torsion axis, where the screen's motion was small.

Symmetry was important in assigning the correct triplet of indices $(j k l)$ to each of the two observed modes. We first assumed symmetrical placement of the oscillator and the fixed electrodes. We also assumed the oscillator's velocity was significant in only the $x$-direction. Location of the oscillator in the plane $x=0$ immediately eliminated from consideration modes with even $j$ because they have $u=0$ in this plane. It also eliminated modes with even $k$ or odd $l$ because their symmetries gave no net coupling to the screen. Thus, only modes such as (112), (114), ..., (132), (134), ..., (312), (314), ..., etc. were considered. These considerations are illustrated in Figure 8.

Of the modes allowed by symmetry, some, such as (114), were eliminated because a superposition of the oscillator's dimensions on the nodal map indicated approximate cancellation of the net torque on the oscillator. (See Figure 


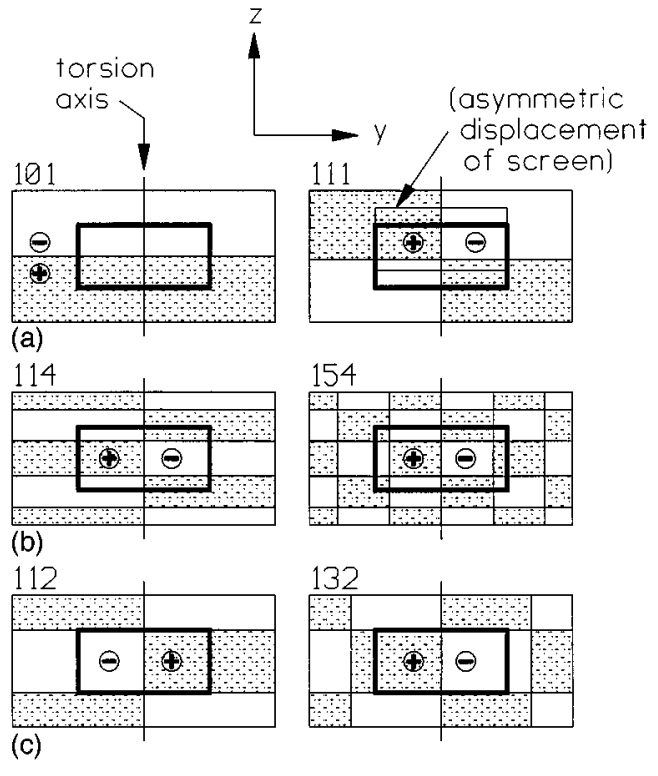

FIG. 8. Symmetry and geometry determined which internal wave modes coupled to the oscillating screen. The cell is in the horizontal orientation and the screen's location in the $y z$ plane is indicated by the rectangular outline. (a) Examples of modes whose symmetry did not allow coupling to oscillating screen. [A slight vertical displacement of the oscillator allowed coupling to mode (111).] (b) Examples of modes allowed by symmetry but discouraged by geometrical cancellation of torques on the screen. (c) Examples of modes allowed by both symmetry and geometry.

8.) Finally, modes with a large index were eliminated because their net coupling to the screen was small. From the above reasoning, the lower observed mode was identified as (112).

The other observed mode was identified as (111) even though (111) has odd $l$ and thus would not be excited in a perfectly symmetrical cell. The identification with (111) was considered because the calculated frequencies of modes with even $l$, such as (112) and (132), could not even qualitatively match the observed temperature dependence. The identification with (111) was consistent with the screen's asymmetric location. Careful measurement of the screen's position within the cell showed a $0.7 \mathrm{~mm}$ vertical displacement from the cell's center, or about $19 \%$ of the screen's half-height. This asymmetry allowed significant coupling between the screen's motion and the (111) mode.

Figure 2 compares the frequencies measured in the horizontal cell with the frequencies calculated numerically for the modes (111) and (112). The calculated frequencies used the physically correct density profiles derived from Eqs. (1). With no adjustable parameters, the calculations successfully describe the measured frequencies to within $15 \%$.

More importantly, the calculations match the differing temperature dependences of the two modal frequencies. The nonmonotonic temperature dependence of mode (112) can be understood by referring to Figure 9a. This mode's flow field has little vertical component near the cell's middle height, where the density gradient is large near $T_{c}$. Thus, the restoring force and associated frequency are smaller than if the density gradient were uniform. In contrast, mode (111) has
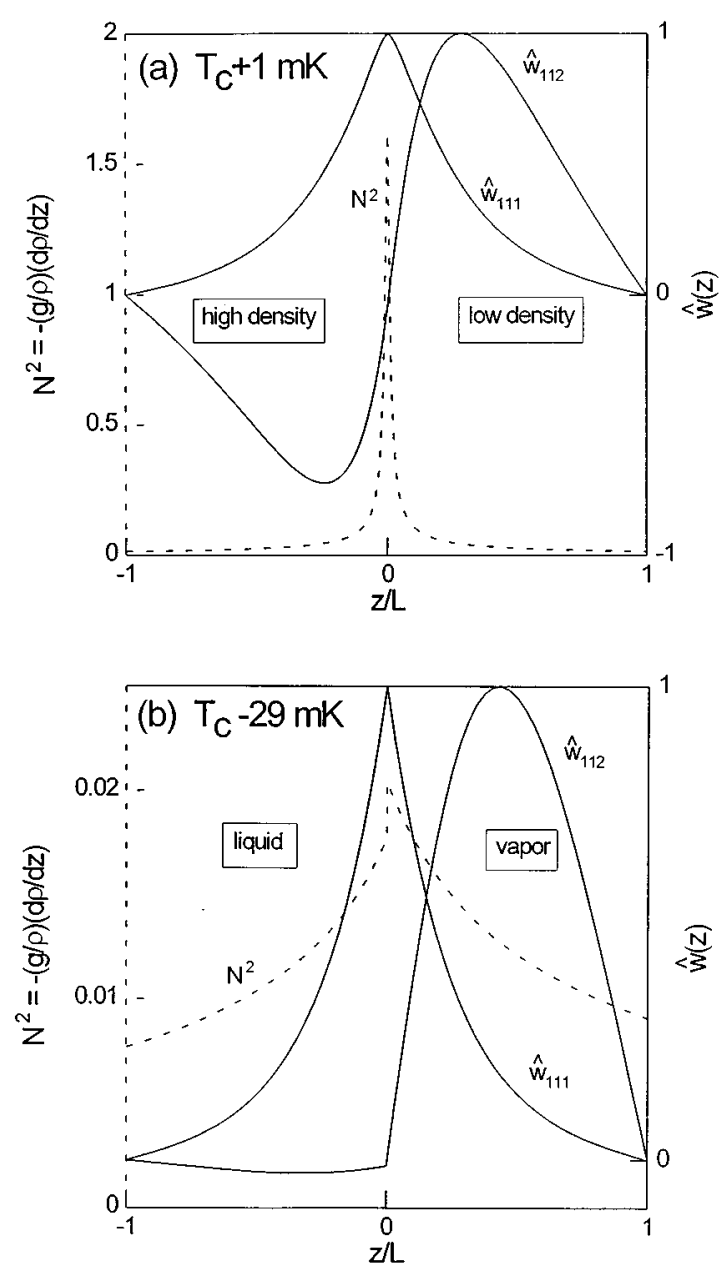

FIG. 9. Plots of the squared Brunt-Väisälä frequency $N^{2}$ and the vertical component of the velocity $\hat{w}$ calculated as a function of the normalized height. (a) At $T_{c}+1 \mathrm{mK}$, mode (111) is nearly symmetric and strongly samples the steep density gradient near the cell's midplane. In contrast, mode (112) has little vertical velocity near the midplane. (b) At $T_{c}-29 \mathrm{mK}$, mode (112) is confined almost entirely to the upper, vapor half of the cell, and thus its frequency is determined chiefly by the average value of $N^{2}$ in that region.

significant flow near $z=0$, and thus it can sample the density gradient peak more effectively.

\section{B. Vertical cell}

Figure 10 shows the results of measurements taken when the cell's axis was vertical and the screen's torsion axis was horizontal. In this orientation, the highest frequencies were the same as in the horizontal orientation. However, in contrast to the horizontal orientation, there were as many as five modes at a single temperature, including at least one lowfrequency mode near $0.6 \mathrm{~Hz}$ with very little temperature dependence. The close spacing of modes complicated the observations, and beating with a nearby mode was seen occasionally. The designations "strong" and "weak" on Figure 10 are qualitative measures of the excited mode's amplitude.

We approximated the cell's geometry by a rectangular box whose dimensions differed from those used for the horizontal orientation only in the interchange of the values of $b$ 


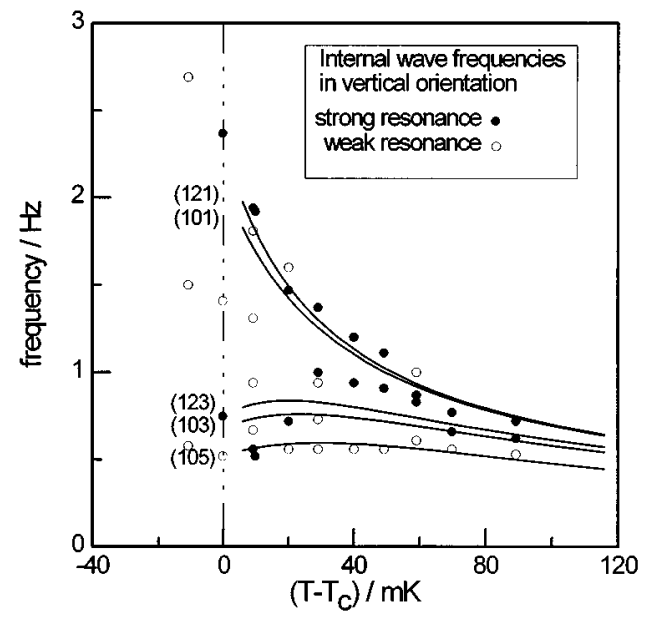

FIG. 10. Internal wave frequencies measured (circles) and calculated (lines) with the cell's axis held vertical. As many as five modes could be seen at one temperature, consistent with the greater number of modes allowed by symmetry than for the horizontal orientation. "Strong", and "weak" indicate the excited mode's observed amplitude.

and $2 L$, consistent with the rotated orientation. As with the horizontal cell, symmetry suppressed certain modes, and thus only modes such as (101), (103), .., (121), (123), .., etc. were considered. However, fewer of the modes allowed by symmetry were eliminated by approximate cancellation of the net torque on the oscillator. Thus, at least four modes, (101), (103), (121), and (123), seemed highly likely. This is consistent with greater number of observed modes observed in the vertical cell.

Figure 10 shows the frequencies for these four modes calculated from the actual density profile by the pseudospectral collocation method. The frequencies calculated for modes (101) and (121) describe the highest frequency data. As was the case for the horizontal cell, the oscillator's asymmetric location allowed coupling to the (111) mode. However, modes with similar vertical dependence and thus similar temperature dependence [(101) and (121)] were allowed, even without the asymmetry. Thus mode (111) was not included in Figure 10. However, because the other modes do not describe the lowest frequency data, the frequency calculated for mode (105) is shown also. This mode was allowed by the cell's symmetry, and its calculated frequency shows the same weak temperature dependence observed at the lowest frequencies.

\section{Discrepancies between the calculated and experimental frequencies}

In the cell's horizontal orientation, the measured frequencies are typically $10 \%$ higher than the numerically calculated frequencies. We considered three possible causes for this discrepancy. The first was the contribution of the oscillator's stiffness to that of the combined fluid-oscillator system. Knowledge of the oscillator's resonance frequency in vacuum, the viscous coupling between the oscillator and the fluid, and the ratios of the kinetic energies stored in the os- cillator and in the fluid allowed us to quantify this effect. The calculated increase in $\omega$ was only $1 \%$, insufficient to explain the discrepancy.

The second possible cause was error in the parameters $k$ or $a$ used in the scaling equation of state for xenon, Eqs. (1). For example, $\omega \propto \sqrt{k}$ is approximately true both above and below $T_{c}$; thus, a $20 \%$ increase of $k$ over the experimental value would decrease the discrepancy to the level of uncertainty in $\omega$. One check on the accuracy of the parameters $k$ and $a$ comes from the assumption of two-scale-factor universality near the critical point, which leads to the prediction of a dimensionless ratio proportional to the product $a k$. Sengers and Moldover ${ }^{26}$ made this comparison for xenon and found agreement between the experimental and theoretical values for $a k$. However, the experimental uncertainties in their comparison do not exclude the possibility of a systematic error in $k$ of $20 \%$.

The third possible cause is the difference between the ideal geometry of the rectangular box used in our calculations and the actual geometry of the cell used in the experiment. The rectangular box modeled the cell's central volume, defined by the $7.6 \mathrm{~mm}$ gap between the stationary electrodes. However, this volume was actually connected to smaller side volumes between the electrodes and the cell's walls. Because these side volumes had gaps less than $6 \mathrm{~mm}$ wide, their characteristic internal wave frequencies were higher than those for the central volume. Thus, coupling between the side volumes and the central volume may have caused the discrepancy.

\section{Comparison of the analytical to the numerical results}

The analytically calculated special cases, where the density profile is approximated by simple functions, give physical insight to the numerical results based on the actual density profile. Figure 5 shows that, far from $T_{c}$, these approximations yield frequencies which agree with those calculated from the actual density profile.

At temperatures above $T_{c}+60 \mathrm{mK}$, the sample's density gradient was approximately linear in $z$. Approximation of $\rho(z)$ by an exponential allowed use of the simple expression Eq. (10), which gave internal-wave frequencies similar to the numerical solution. For this special case, we derived the characteristic Brunt-Väisälä frequency $N$ from the maximum density gradient at the cell's middle. At temperatures closer to $T_{c}$, the exponential model for $\rho(z)$ was no longer valid, as demonstrated in Figure 5 by the disagreement with the frequencies calculated from the actual density profile.

At temperatures below $T_{c}-5 \mathrm{mK}$, the density profile was approximately two layers of constant density separated by a sharp interface. This allows use of Eq. (20) to calculate the frequency of mode (111). As shown in Figure 5, this approximation is valid much closer to $T_{c}$ than the exponential approximation used above $T_{c}$. The success of the twolayer model can be understood by examining Figure 9b, which contrasts the eigenfunctions $\hat{w}(z)$ of modes (111) and (112) derived from the actual density profile at $T_{c}-29 \mathrm{mK}$. For mode (111), the form of $\hat{w}(z)$ is very nearly the same as 
the hyperbolic functions which appear in the two-layer model. [See Eq. (19).]

This two-layer model with constant densities cannot explain mode (112) below $T_{c}$, which, as shown in Figure 9b, has vertical motion predominantly in the vapor region. Thus, (112) is approximately an internal wave mode confined to the vapor region, whereas (111) is approximately an interface mode. Neglect of the liquid layer entirely and assumption of an exponential density profile (constant $N^{2}$ ) within the vapor phase allows for the calculation of mode (112)'s frequencies from Eq. (10). For this special case, we derived the characteristic Brunt-Väisälä frequency $N$ from the height-averaged density gradient in the cell's upper half. Figure 5 shows that this gives a good approximation of the temperature dependence calculated for mode (112) from the actual density profile.

\section{CONCLUSIONS}

In our measurements, by cooling the temperature only $140 \mathrm{mK}$, we changed the xenon's density profile from linear, to sigmoid, to discontinuous. In the horizontal cell, the resulting large increase in the density profile's gradient increased the frequency of mode (111) by a factor of four. The large increase in the density profile's nonlinearity increased the separation of modes (111) and (112), so that their frequency ratio increased from 1.2 to 5.2. Our calculations of the internal wave modal frequencies, based on the actual density profile expected from xenon's equation of state, agreed to within $15 \%$ with the measured values.

The symmetry of the internal wave modes was important because it restricted which fluid modes could couple to the oscillating screen. For the cell's horizontal orientation, this restriction greatly simplified the observations: only two, well-separated modes were observed.

The modal symmetry was also important in understanding the different temperature dependences of the observed modes. Mode (111), with a large vertical amplitude near the cell's middle height, evolved into an interface mode as the temperature was cooled below $T_{c}$. In contrast, mode (112) evolved into an internal wave mode trapped in the cell's upper, vapor half. Analytical calculations assisted this understanding. The special case of an exponential density profile approximated the frequencies of modes (111) and (112) far above $T_{c}$, and, when applied to the cell's upper half only, it approximated the frequencies of mode (112) below $T_{c}$. The special case of two layers of constant density explained the frequencies of mode (111) below $T_{c}$.

The analytic special cases could not explain the differing temperature dependences of (111) and (112) just above $T_{c}$, especially (112)'s nonmonotonic temperature dependence. However, Figure 9 provides physical understanding by plotting the amplitudes of both modes and $N^{2}(z)=-(\mathrm{g} /$ $\rho)(d \rho / d z)$. The two modes differ greatly in their sampling of $N^{2}$ : at $T_{c}+1 \mathrm{mK}$, the region of large $N^{2}$ is too narrow to affect the (112) mode.

Our results suggest two further applications of internal wave modes in nearly critical fluids. First, the fluid's equation of state could be improved. The parameter $k$ used in the scaling equation of state came from optical measurements of the static density profile. ${ }^{19}$ Measurements of the frequencies of internal-wave modes in a well-defined container would offer an independent check on the optical measurements of $k$ and would perhaps have greater accuracy. Second, nearly critical fluids may be superior to stratified salt-water for some investigations of internal waves. A wide variety of density profiles is available not only by adjusting the temperature but also by adjusting the sample's average density. Because these profiles are the fluid's equilibrium state, profile disturbances caused by the measurement itself, breaking of waves for example, are eliminated simply by waiting for equilibration.

\section{ACKNOWLEDGMENTS}

This investigation resulted from Professor John Reppy's suggestion of using the coupled oscillator method to observe the viscometer's internal wave modes. Michael Moldover was a collaborator in the oscillator's design and instrumentation, and he first made the suggestion of a mode having symmetry similar to (112). Useful conversations with Hacène Boukari and Greg Zimmerli are acknowledged. Work by R. Berg was sponsored in part by NASA under Contract No. C-32014-C. Work by M. Lyell was partially supported by a sabbatical leave from West Virginia University. Work by G. McFadden was sponsored in part by NASA under Contract No. C-82008-B.

${ }^{1}$ H. Lamb, Hydrodynamics (Dover, New York, 1945).

${ }^{2}$ L. Prandtl, Essentials of Fluid Dynamics (Blackie, London, 1952).

${ }^{3}$ L. D. Landau and E. M. Lifshitz, Fluid Mechanics (Pergamon, New York, 1959).

${ }^{4}$ D. J. Tritton, Physical Fluid Dynamics, 2nd ed. (Oxford University Press, New York, 1988).

${ }^{5}$ P. K. Kundu, Fluid Mechanics (Academic, New York, 1990).

${ }^{6} \mathrm{C}$. Eckart, Hydrodynamics of Oceans and Atmospheres (Pergamon, New York, 1960).

${ }^{7}$ O. M. Phillips, The Dynamics of the Upper Ocean (Cambridge University Press, Cambridge, 1969).

${ }^{8}$ J. S. Turner, Buoyancy Effects in Fluids (Cambridge University Press, Cambridge, 1973).

${ }^{9}$ J. Lighthill, Waves in Fluids (Cambridge University Press, Cambridge, 1978).

${ }^{10}$ R. G. Rehm, "A survey of selected aspects of stratified and rotating fluids,' J. Res. Natl. Bur. Stand. 80B, 353 (1976).

${ }^{11}$ H. Boukari (private communication, 1994).

${ }^{12}$ M. Sh. Giterman, "Study of the critical state of a liquid using gravitational waves,' Sov. Phys. Dokl. 12, 1141 (1968).

${ }^{13} \mathrm{~V}$. D. Khait, "On gravitational waves in a gas near the critical point for the gas-liquid transition,'” Sov. Phys. JETP 30, 177 (1970).

${ }^{14}$ M. Sh. Giterman and V. A. Shteinberg, "Internal gravitational waves and convectional instability in liquids,' Mekh. Zhi. Gosta 7, 55 (1972); English translation in Fluid Dyn. 7, 238 (1972).

${ }^{15}$ R. F. Berg and M. R. Moldover, Science Requirements Document (1993), report to Space Experiments Division, NASA Lewis Research Center, Cleveland, OH 44135.

${ }^{16}$ R. F. Berg, "Hydrodynamic similarity in an oscillating-body viscometer," Int. J. Thermphys. 16, 1257 (1995).

${ }^{17}$ J. T. Ho and J. D. Litster, "Faraday rotation near the ferromagnetic critical temperature of $\mathrm{CrBr}_{3}$,'” Phys. Rev. B 2, 4523 (1970).

${ }^{18}$ M. R. Moldover, J. V. Sengers, R. W. Gammon, and R. J. Hocken, “Gravity effects in fluids near the gas-liquid critical point,', Rev. Mod. Phys. 51, 79 (1979)

${ }^{19}$ R. J. Hocken and M. R. Moldover, "Ising critical exponents in real fluids: An experiment,'’ Phys. Rev. Lett. 37, 29 (1976).

${ }^{20} \mathrm{P}$. C. Hohenberg and M. Barmatz, "Gravity effects near the gas-liquid critical point,' Phys. Rev. A 6, 289 (1972).

${ }^{21}$ J. Thoen and C. W. Garland, "Sound absorption and dispersion as a func- 
tion of density near the critical point of xenon,', Phys. Rev. A 10, 1311 (1974).

${ }^{22}$ D. Gottlieb, M. Y. Hussaini, and S. A. Orszag, in Spectral Methods for Partial Differential Equations, edited by R. G. Voigt, D. Gottlieb, and M. Y. Hussaini (Society for Industrial and Applied Mathematics, Philadelphia, 1984), p. 1

${ }^{23}$ H. B. Keller, Numerical Solutions of Two Point Boundary Value Problems, Regional Conference Series in Applied Mathematics (SIAM, Phila- delphia, 1976), Vol. 24.

${ }^{24}$ M. R. Scott and H. A. Watts, "Computational solution of linear two-point boundary value problems via orthonormalization,', SIAM J. Num. Anal. 14, 40 (1977).

${ }^{25}$ I. G. Currie, Fundamental Mechanics of Fluids, 2nd ed. (McGraw-Hill, New York, 1993).

${ }^{26}$ J. V. Sengers and M. R. Moldover, "Two-scale universality near the critical point of fluids,' Phys. Lett. A 66, 44 (1978). 